\title{
A MATHEMATICAL STUDY OF A BISTABLE NEMATIC LIQUID CRYSTAL DEVICE
}

\author{
F. P. DA COSTA \\ Departamento de Ciências Exactas e Tecnológicas, \\ Universidade Aberta, Rua Fernão Lopes, 9, 2 Dto, \\ P-1000-132 Lisboa, Portugal \\ and \\ Center for Mathematical Analysis, Geometry and Dynamical Systems, \\ Instituto Superior Técnico, TU Lisbon, \\ Av. Rovisco Pais, 1 , \\ P-1049-001 Lisboa, Portugal \\ fcosta@univ-ab.pt \\ M. GRINFELD* and N. J. MOTTRAM ${ }^{\dagger}$ \\ Department of Mathematics, University of Strathclyde, \\ 26 Richmond Street, Glasgow G1 $1 X H$, UK \\ *mg@maths.strath.ac.uk \\ ${ }^{\dagger}$ nigel.mottram@strath.ac.uk

\section{J. T. PINTO} \\ Department of Mathematics and Center for Mathematical Analysis, \\ Geometry and Dynamical Systems, \\ Instituto Superior Técnico, TU Lisbon, \\ Av. Rovisco Pais, 1, \\ P-1049-001 Lisboa, Portugal \\ jpinto@math.ist.utl.pt \\ Received 5 June 2006 \\ Revised 18 April 2007 \\ Communicated by K. R. Rajagopal
}

\begin{abstract}
We consider a model of a bistable nematic liquid crystal device based on the EricksenLeslie theory. The resulting mathematical object is a parabolic PDE with nonlinear dynamic boundary conditions. We analyze well-posedness of the problem and global existence of solutions using the theory developed by Amann. Furthermore, using phaseplane methods, we give an exhaustive description of the steady state solutions and hence of the switching capabilities of the device.
\end{abstract}

Keywords: Nematic liquid crystals; bistable device; switching; parabolic PDEs; dynamic boundary conditions.

AMS Subject Classification: 35B32, 35K57, 76A15, 82D30 


\section{Introduction}

The liquid crystalline state of matter exists, for certain materials, in a temperature range between the solid crystalline phase and the isotropic liquid phase. Materials that exhibit such a phase usually consist of rod-like (or disc-like) molecules. In the liquid crystal phase a molecular self-organisation occurs to produce orientational order where the rod or disc-like molecules prefer to lie approximately parallel to each other. In some materials there may also exist an amount of positional ordering (i.e. into two-dimensional layers) of the molecules. This orientational/positional ordering leads to a number of crystal-like properties, whilst the material remains in the fluid phase, hence the name liquid crystal. The orientational order within the liquid crystal allows us to define an anisotropic axis, the axis of rotational symmetry. This anisotropic axis, the average molecular direction at that point in the material, is a macroscopic variable, called the director $\mathbf{n}$ (a unit vector). The director may vary in space (and change with time) to create director distortion structures which increase the stored elastic energy of the system. The organic molecules that form the liquid crystal material may contain permanent or induced electric dipoles. The director can therefore be influenced by the application of an electric field.

The existence of an anisotropic axis in the system means that the liquid crystal is birefringent, with light polarised along the long molecular axis travelling at a different speed to light polarised along the short molecular axis. The birefringence and the consequential phase retardation of light means that liquid crystals can be used in a display device. By sandwiching the liquid crystal between two glass or plastic substrates, using optical elements such as polarisers, and an electric field which changes the orientation of the director, the liquid crystal can be switched so that the device can allow light to be transmitted through the device or extinguished. Using patterned electrodes to define pixels and colour filters, a flat (compared to a cathode ray tube) display system can be produced: a liquid crystal display (LCD).

The versatility, portability and space saving aspects of LCDs means that they are now ubiquitous. From small simple displays on calculators and electrical appliances to more complex video displays on mobile phones, portable audio and video devices and televisions, the manufacture of LCDs remains a growth industry.

Most current LCDs are monostable; there is a single stable director configuration which is changed when an electric field is applied. In these types of displays, to maintain an image, a voltage needs to be constantly applied. This is not an ideal situation for portable devices which have a limited battery power supply. In recent years a number of bistable liquid crystal devices have been developed which have two stable base states. In this case a voltage need only be applied when a pixel needs to be changed and a static image needs not drain the power supply. This would be particularly useful for future applications such as e-paper, a display device onto which information (i.e. a newspaper) could be downloaded.

A number of bistable liquid crystal devices use a nematic liquid crystal, which has orientational order (and therefore a director $\mathbf{n}$ ) but no positional order, and 
a patterned substrate which allows two types of director orientation. This bistable surface creates two possible director configurations within the bulk of the liquid crystal. A simple model of two particular bistable LCDs (the $\mathrm{ZBD}^{6}$ and $\mathrm{PABN}^{16}$ displays) has been proposed by Davidson and Mottram. ${ }^{8,9}$ In the model the complicated structured bistable substrate is replaced by a simpler planar substrate which exhibits two preferred director orientations. In this way the intrinsically 2D or $3 \mathrm{D}$ nature of the $\mathrm{ZBD}$ and $\mathrm{PABN}$ devices is replaced by a $1 \mathrm{D}$ mathematical model.

In this paper we investigate various mathematical issues relating to such a bistable display. The plan of this paper is as follows. After a brief derivation of the equations in Sec. 2, we deal with issues of well-posedness and long-time behaviour of solutions in Sec. 3. Problems of multiplicity and stability of stationary solutions which have direct bearing on the ability of device to be bistable and to switch between the stable states are investigated in Sec. 4. It turns out that there are two different cases to consider, based on the sign of the dielectric anisotropy of the material. In both cases it is shown that the ability to switch is crucially dependent on the flexoelectric properties of the material. Finally, in Sec. 5 we consider the behaviour of the device under the influence of rapidly oscillating electric field using methods of averaging theory.

In order to model the director dynamics within this device we will use the Ericksen-Leslie formulation ${ }^{23}$ which considers the balances of mass, linear momentum and angular momentum together with constitutive equations for the free energy and dissipation which are assumed to be functions of the director and gradients in the director. Such an approach is only one of the many possible theories which have been used to model liquid crystal materials. For instance, other theories have considered the molecular order as a dependent variable and the dissipation and free energy is then written in terms of an order parameter (see, for instance, Ref. 22). An alternative approach, which determines the evolution of the director configuration through a maximal rate of dissipation, ${ }^{20}$ has also been formulated which removes the need to prescribe the director at the substrates. In our system the physical surface alignments (through surface layer treatment and substrate morphology) lead to physically realistic boundary conditions and we can therefore utilise the EricksenLeslie equations. For more details on the physics of the device and a numerical investigation of its behaviour, see Refs. 9 and 8 .

\section{Derivation of the Model}

The model proposed by Davidson and Mottram assumes that the system is invariant in any direction parallel to the substrates. The director therefore only varies across the liquid crystal layer, in the $z$-direction. The director is therefore a variable of $z$ and time $t$. Since the liquid crystal is restricted by the substrates in the $z$-direction and we assume invariance in the $x y$-plane, there will be no fluid flow. The nematic molecules, and therefore the director, will simply rotate with no bulk movement of 
the fluid. These simplifications are equivalent to assuming that the only dissipative process within the liquid crystal is due to the director rotation and not to viscous dissipation. This assumption is not always valid but in the present situation a number of studies have shown that the director reorientation due to fluid flow is a transient effect which essentially renormalises the relaxation coefficient of the liquid crystal. $^{23}$

To model this type of bistable device, it is appropriate to fix the director at the top substrate to be vertical (in the $z$-direction) and allow the lower substrate to attain a minimum energy configuration when the director is either parallel or perpendicular to the substrate. The rigid anchoring at the top substrate can be achieved with the use of a surfactant and the strength of this anchoring has been shown to be sufficient to assume that the director remains perpendicular to the substrate for all reasonable electric field strengths. ${ }^{21}$ The bistable nature of the lower substrate is particular to the device we are considering. Whilst the exact orientations of the director at the substrate may not be parallel and perpendicular in the real devices, we use this example of bistability to build up a qualitative picture of the switching effects. As shown in Fig. 1, this type of anchoring creates the possibility of two different configurations of the liquid crystal layer, the vertical (V) state and the hybrid aligned nematic (HAN) state. It is the ability of the device to switch between these two stable states that we are interested in investigating. Figure 1 also shows the director angle $\theta$ which defines the director as $\mathbf{n}(z, t)=$ $(\cos \theta(z, t), 0, \sin \theta(z, t))$.

The interaction between liquid crystal molecules and an electric field in standard liquid crystal displays occurs through the dielectric effect. When an electric field is applied, a molecular dipole is induced along the molecular axis with the largest dielectric susceptibility. This induced dipole then orients with the electric field direction. In a material with a positive dielectric anisotropy, the largest susceptibility is along the long molecular axis and therefore the director prefers to align parallel to an electric field. For such materials this would mean that we should expect a HAN to $\mathrm{V}$ switch to be relatively easy. For a material with a negative
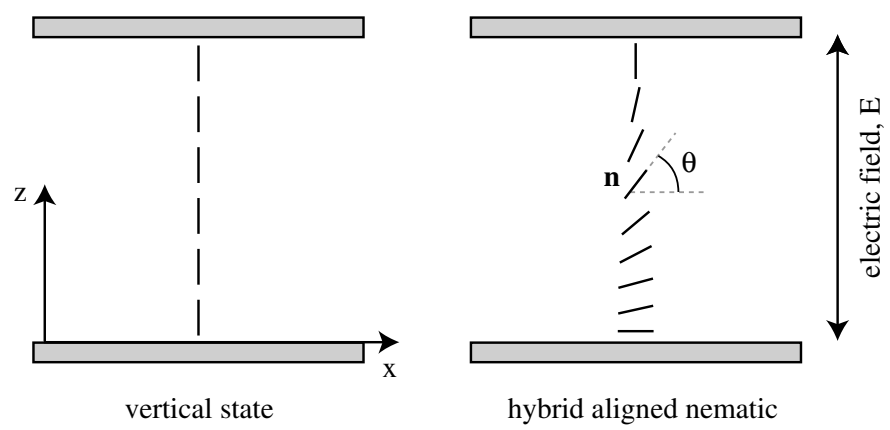

Fig. 1. Possible states of the bistable nematic device. 
dielectric anisotropy, the largest susceptibility occurs along the short molecular axis and therefore the director would align in a direction perpendicular to that of the electric field and would make a V to HAN switching relatively easy. However, a material has either a positive or a negative dielectric anisotropy. For any material only switching one way can be achieved with this dielectric effect.

To enable both switches to be possible, we need a material which differentiates between positive and negative $E$. One possibility is flexoelectricity. Such an effect occurs in materials which consists of molecules containing both a permanent dipole and a shape anisotropy such as the pear-shaped molecules in Fig. 2. In this case an electric field will align the permanent dipoles and induce a splay distortion in the molecular configuration. The crucial point is that electric fields in opposite directions cause different distortions and may therefore be able to enable switching between two stable states.

In order to model the director dynamics within this device we will use the Ericksen-Leslie equation for the rotation of the director, neglecting flow effects. ${ }^{23}$ As mentioned above this assumption implies that we only consider the dissipation due to director rotation and not from viscous effects. This will be equivalent to a gradient flow approach where the free energy is

$$
F=\int_{0}^{d}\left(\mathcal{F}_{e}+\mathcal{F}_{d}+\mathcal{F}_{f}\right) d z+F_{s}
$$

and the free energy densities $\mathcal{F}_{e}, \mathcal{F}_{d}$ and $\mathcal{F}_{f}$ represent contribution from elastic distortions, the dielectric effect, and the flexoelectric coupling. The energy $F_{s}$ is the surface free energy at the bistable surface $z=0$. Standard expressions for the free energy densities are used,

$$
\begin{aligned}
& \mathcal{F}_{e}=\frac{K}{2}\left((\nabla \cdot \mathbf{n})^{2}+|\nabla \times \mathbf{n}|^{2}\right), \\
& \mathcal{F}_{d}=-\frac{\epsilon_{0} \Delta \epsilon}{2}(\mathbf{E} \cdot \mathbf{n})^{2} \\
& \mathcal{F}_{f}=-\left(e_{11}(\nabla \cdot \mathbf{n}) \mathbf{n}+e_{33}((\nabla \times \mathbf{n}) \times \mathbf{n})\right) \cdot \mathbf{E},
\end{aligned}
$$
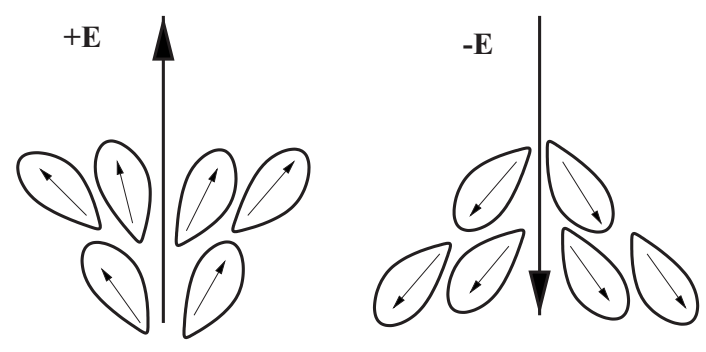

Fig. 2. The flexoelectric effect. 
where $K$ is the isotropic elastic constant; $\Delta \epsilon$ is the dielectric anisotropy coefficient which may be positive or negative (as mentioned above), $\epsilon_{0}$ is the permittivity of free space; $\mathbf{E}$ is the electric field; $e_{11}$ and $e_{33}$ are flexoelectric coefficients.

We will assume that the surface energy due to the lower substrate morphology is

$$
F_{s}=4 W_{0}\left(\mathbf{n} \cdot \mathbf{n}_{s}\right)^{2}\left|\mathbf{n} \times \mathbf{n}_{s}\right|^{2},
$$

where $W_{0}>0$ is the anchoring strength and $\mathbf{n}_{s}=(0,0,1)$ is the surface normal, so that there are minima of the energy when the director is parallel to $\mathbf{n}_{s}$ and perpendicular to $\mathbf{n}_{s}$. The upper surface will assume to induce infinitely strong anchoring of the director so that $\theta=\pi / 2$ at $z=d$.

In this paper we will assume that the director remains in the $x z$-plane so that $\mathbf{n}(z, t)=(\cos \theta(z, t), 0, \sin \theta(z, t))$ and that the electric field $\mathbf{E}=(0,0, E)$ is related to the voltage across the plates $V$ by $E=-V / d$ rather than being found from Maxwell's equations. This means that the free energy reduces to

$$
F=\int_{0}^{d} \frac{1}{2}\left\{K\left(\theta_{z}\right)^{2}-\epsilon_{0} \Delta \epsilon E^{2} \sin ^{2} \theta-2 E_{13} E \sin (2 \theta) \theta_{z}\right\} d z+W_{0} \sin ^{2}(2 \theta(0, t)),
$$

where $E_{13}=\left(e_{11}+e_{33}\right) / 2$ is the average flexoelectric coefficient. For the subsequent analysis we will assume that $E_{13}>0$ for simplicity, although the case $E_{13}<0$ is simple to consider by exchanging the sign of $V$, since the transformation $E_{13} \rightarrow$ $-E_{13}, V \rightarrow-V$ leaves the system of equations unchanged. It should also be noted that since the flexoelectric term in (2.6) can be integrated to give a surface term, $-E_{13} E \cos (2 \theta(0, t)) / 2$, then for $E>0$, this contribution is minimised by $\theta(0, t)=0$ and for $E<0$, by $\theta(0, t)=\pi / 2$. The difference in behaviour of the surface anchoring for positive and negative voltages will enable the bistable device to be switched backwards and forwards between the two stable states.

With the free energy in (2.6) the dynamic equations are derived from the balance of the rate of dissipation and the gradient of the free energy:

$$
\left\{\begin{aligned}
\gamma_{1} \theta_{t} & =K \theta_{z z}+\frac{1}{2} \epsilon_{0} \Delta \epsilon E^{2} \sin (2 \theta), & & \text { for }(t, z) \in \mathbb{R}^{+} \times(0, d), \\
\gamma_{s} \theta_{t} & =K \theta_{z}-2 W_{0} \sin (4 \theta)-E_{13} E \sin (2 \theta), & & \text { when }(t, z) \in \mathbb{R}^{+} \times\{0\}, \\
\theta & =\frac{\pi}{2}, & & \text { when }(t, z) \in \mathbb{R}^{+} \times\{d\} .
\end{aligned}\right.
$$

Here $\gamma_{1}$ is the director rotation viscosity and $\gamma_{s}$ is the surface relaxation coefficient related to a surface dissipation effect. The thermodynamic restrictions on material parameters which ensure the non-negativity of the free energy and the dissipation are $K>0, \gamma_{1}>0$ and $\gamma_{s}>0 .{ }^{23}$ In this system it is important to add such a surface dissipation term because the substrate at $z=0$ is actually mimicking a complicated structured morphology which involves both the surface anchoring effects and the dissipative liquid crystal material in the vicinity of the substructure. It is clearly the dynamical (nonlinear) boundary condition in (2.7) that makes this into a mathematically nontrivial problem. 
As discussed in the Introduction, we shall consider the situation for both constant $E$ (changing it adiabatically) and for $E$ a fast-oscillating periodic function of time.

In order to bring out the mathematical structure of the problem in a clearer way we non-dimensionalise the system by considering the following variables

$$
x:=\frac{z}{d}, \quad \tau:=\frac{K}{\gamma_{1} d^{2}} t, \quad u(\tau, x):=\theta\left(\tau \frac{\gamma_{1} d^{2}}{K}, x d\right),
$$

and introducing the parameters

$$
\begin{gathered}
\nu=\frac{\gamma_{s}}{\gamma_{1} d}, \quad \beta=\frac{2 W_{0} d}{K}, \quad \sigma=\operatorname{sgn}(\Delta \epsilon), \\
\kappa=\frac{E_{13}}{\sqrt{\frac{\epsilon_{0}|\Delta \epsilon|}{2} K}}, \quad \eta=\sqrt{\frac{\epsilon_{0}|\Delta \epsilon|}{2} \frac{d^{2}}{K}} E .
\end{gathered}
$$

With these definitions, system (2.7) is transformed into

$$
\left\{\begin{aligned}
u_{\tau} & =u_{x x}+\sigma \eta^{2} \sin (2 u), & & \text { for }(\tau, x) \in \mathbb{R}^{+} \times(0,1), \\
\nu u_{\tau} & =u_{x}-\beta \sin (4 u)-\kappa \eta \sin (2 u), & & \text { when }(\tau, x) \in \mathbb{R}^{+} \times\{0\}, \\
u & =\frac{\pi}{2}, & & \text { when }(\tau, x) \in \mathbb{R}^{+} \times\{1\} .
\end{aligned}\right.
$$

\section{Well-Posedness and Asymptotic Behavior}

Problems with boundary conditions such as (2.8) have been discussed in the literature in the last 15 years. ${ }^{1,3,10-12,18}$ The main scheme of proving global existence for parabolic problems with nonlinear boundary conditions follows ${ }^{1}$ see also Ref. 17 for an accessible introduction to Amann's theory. A specific application of this theory to parabolic problems with dynamical boundary conditions was given in Refs. 10 and 11. In Ref. 12, also based on the same functional approach, further results on the large time behavior of solutions are obtained for a specific type of semilinear problem. Since in our case we impose a Dirichlet type boundary condition on a part of the boundary, which is not considered in Refs. 10-12, the results in these works cannot be directly applied to our case. However, we use its framework to extend those results to our type of boundary conditions. In this section we give an outline of that approach.

Let $\Omega \subset \mathbb{R}^{n}$ be an open bounded set with smooth boundary $\Gamma_{0}=\Gamma_{1} \cup \Gamma_{2}$, such that $\Gamma_{1}$ and $\Gamma_{2}$ are separated sets. Let $\mu_{i}$ be the trace operator on $\Gamma_{i}, i=1,2$ and $\nu$ a positive constant. Then the problem we are considering is of the form

$$
\begin{cases}\partial_{t} u+\mathcal{A} u=f(\cdot, u, t) & \text { in } \Omega \times(0, \infty) \\ \partial_{t} v+\mathcal{B}_{1} u=g(\cdot, v, t) & \text { on } \Gamma_{1} \times(0, \infty), \\ \nu \mu_{1} u=v & \text { on } \Gamma_{1} \times(0, \infty), \\ \mu_{2} u=0 & \text { on } \Gamma_{2} \times(0, \infty),\end{cases}
$$


and the (compatible) initial conditions

$$
\begin{cases}u(z, 0)=u_{0}(z) & z \in \Omega, \\ v(z, 0)=v_{0}(z)=\nu \mu_{1} u(z, 0) & z \in \Gamma_{1} .\end{cases}
$$

Here $\mathcal{A}$ is a uniformly strongly elliptic operator of the form

$$
\mathcal{A} u:=-\partial_{i}\left(a_{i j} \partial_{j} u\right)+a_{i} \partial_{i} u+a_{0} u,
$$

with $a_{i j}, a_{i}$ and $a_{0}$ being smooth functions of $x \in \bar{\Omega}$, and $\mathcal{B}_{1}$ is the boundary operator given by

$$
\mathcal{B}_{1} u:=-\mu_{1}\left(\partial_{\mathbf{n}_{a}} u+b u\right),
$$

where $\mathbf{n}_{a}(y)$ is the conormal at $y \in \Gamma_{1}$ and $b$ is a smooth function of $y \in \Gamma_{1}$. We take $f$ and $g$ to be sufficiently smooth; e.g. $g_{i} \in C^{1,1}\left(\mathbb{R} \times \mathbb{R}_{+}, \mathbb{R}\right)$. By setting $w=(u, v)$, we define the following substitution operators:

$$
\begin{array}{ll}
\hat{f}(w, t)(x):=f(x, u(x), t), & \text { for } x \in \Omega, \\
\hat{g}(w, t)(y):=g\left(y, \mu_{1} u(y), t\right), & \text { for } y \in \Gamma_{1} .
\end{array}
$$

Now, defining $G(w, t):=(\hat{f}(w, t), \hat{g}(w, t))$ and $\mathbb{A} w:=\left(\mathcal{A} u, \mathcal{B}_{1} u\right)$ for $w$ in a suitable space that takes into account the boundary condition on $\Gamma_{2}$ and the condition $\nu \mu_{1} u=v$, we can write (3.1) as the Cauchy problem

$$
w_{t}+\mathbb{A} w=G(w, t), \quad(z, t) \in \Omega \times \mathbb{R}_{+}, \quad w(0)=w_{0},
$$

with an appropriate initial condition $w_{0}$. Here and in the following we do not mention the dependence on $\nu$ in the notation, assuming $\nu$ is fixed from the start.

The operator $\mathbb{A}$ takes

$$
\widetilde{E}_{1}:=\left\{(u, v) \in H_{q, \mu_{2}}^{2}(\Omega) \times B_{q}^{2}\left(\Gamma_{1}\right), v=\nu \mu_{1} u\right\}
$$

into

$$
\widetilde{E}_{0}:=L_{q}(\Omega) \times B_{q}^{1}\left(\Gamma_{1}\right)
$$

Here

$$
H_{q, \mu_{2}}^{2}(\Omega)=\left\{u \in H_{q}^{2}(\Omega), \mu_{2} u=0 \text { on } \Gamma_{1}\right\},
$$

$H_{q}^{2}(\Omega)$ is the Bessel potential space and $B_{q}^{2}\left(\Gamma_{1}\right):=B_{q q}^{s-1 / q}\left(\Gamma_{1}\right)$ is the appropriate Besov space required by the trace theorem.

Let us also define, for each $\vartheta \in I_{q}:=(1 / 2 q, 1 / 2+1 / 2 q)$,

$$
\begin{aligned}
E_{\vartheta} & :=\left\{(u, v) \in H_{q, \mu_{2}}^{2 \vartheta}(\Omega) \times B_{q}^{2 \vartheta}\left(\Gamma_{1}\right), v=\nu \mu_{1} u\right\} \\
E_{1-\vartheta}^{\sharp} & :=\left\{(\varphi, \psi) \in H_{q^{\prime}, \mu_{2}}^{2-2 \vartheta}(\Omega) \times B_{q^{\prime}}^{2-2 \vartheta}\left(\Gamma_{1}\right), v=\nu \mu_{1} u\right\} \\
E_{\vartheta-1} & :=\left[E_{1-\vartheta}^{\sharp}\right]^{\prime}, \quad E_{-\vartheta}^{\sharp}:=\left[E_{\vartheta}\right]^{\prime} .
\end{aligned}
$$

Here we are identifying $\left[L_{q}(\Omega) \times L_{q}\left(\Gamma_{1}\right)\right]^{\prime}$ with $L_{q^{\prime}}(\Omega) \times L_{q^{\prime}}\left(\Gamma_{1}\right)$ according to the duality pairing

$$
\langle(\varphi, \psi),(u, v)\rangle:=\langle\varphi, u\rangle+\nu^{-1}\langle\psi, v\rangle_{1},
$$


for $(\varphi, \psi) \in L_{q^{\prime}}(\Omega) \times L_{q}\left(\Gamma_{1}\right)$ and $(u, v) \in L_{q}(\Omega) \times L_{q}\left(\Gamma_{1}\right)$, where $\langle\cdot, \cdot\rangle$ and $\langle\cdot, \cdot\rangle_{1}$ are the standard duality pairing in $L_{q^{\prime}}(\Omega) \times L_{q}(\Omega)$ and $L_{q^{\prime}}\left(\Gamma_{1}\right) \times L_{q}\left(\Gamma_{1}\right)$, respectively. As in Ref. 11 we can prove that

$$
\widetilde{E}_{1} \stackrel{d}{\hookrightarrow} E_{\vartheta} \stackrel{d}{\hookrightarrow} \widetilde{E}_{0} \stackrel{d}{\hookrightarrow} E_{\vartheta-1}
$$

In the next lemma we collect important results concerning the linear operators we need to consider:

Lemma 3.1. Given $\vartheta \in I_{q}, \mathbb{A}$ is closable in $E_{\vartheta-1}$ and calling $\mathbb{A}_{\vartheta-1}$ to the respective closure,

(1) $\operatorname{dom}\left(\mathbb{A}_{\vartheta-1}\right)=E_{\vartheta}$;

(2) there is $\omega>0$ such that $\lambda+\mathbb{A}_{\vartheta-1} \in \operatorname{Iso}\left(E_{\vartheta}, E_{\vartheta-1}\right)$, for all $\lambda \in[\operatorname{Re} \mu \geq \omega]$;

(3) the operator $-\mathbb{A}_{\vartheta-1}$ generates a strongly continuous analytic semigroup on $E_{\vartheta-1}$;

(4) if $1 / 2 q<\vartheta<\eta \leq 1$, then,

$$
\left\|e^{-t \mathbb{A}_{\eta-1}}\right\|_{\mathcal{L}\left(E_{\eta-1}, E_{\vartheta}\right)} \leq C t^{\eta-\vartheta-1} .
$$

The proof of these statements follows the same lines of arguments of Refs. 10 and 11 and, for (4), it can be obtained in an interpolation-extrapolation Banach spaces framework, (see Ref. 10), by using a result in Ref. 2.

Remark. The analyticity of the resulting semigroup will be required in Sec. 5.

Also instrumental for the local existence and uniqueness proof is a "sandwich" result for the complex interpolation of the spaces introduced above. Let $[\cdot ; \cdot]_{\eta}, \eta \in$ $(0,1)$ be the standard complex interpolation functor (for the general theory, see Ref. 4). Then

Lemma 3.2. If $\xi, \vartheta \in I_{q}$ with $\xi<\vartheta$, then setting $\delta:=\vartheta-\xi$,

$$
\left[E_{\vartheta-1} ; E_{\vartheta}\right]_{1-\delta} \stackrel{d}{\hookrightarrow} E_{\xi} \stackrel{d}{\hookrightarrow}\left[E_{\vartheta-1} ; E_{\vartheta}\right]_{1-\frac{\delta}{1-\vartheta}} .
$$

It should be noted that a true reiteration result does not hold (see Refs. 10 and 11). For a proof of this result we can consider an easy modification of the case in Ref. 10 .

The final result of this approach is a local existence theorem. We present first some necessary definitions. We need to consider the semilinear problem (3.3) in a weaker sense. Given $q \geq 2$, fixing $\eta, \vartheta, \in I_{q}$, such that $\vartheta<\eta$, we consider the following Cauchy problem:

$$
w_{t}+\mathbb{A}_{\vartheta-1} w=G(w, t), \quad w(0)=w_{0},
$$

where, with the above hypothesis, the nonlinearity $G$ is proved to be a map from $[0, T] \times E_{\vartheta}$ to $E_{\eta-1}$, which is uniformly Lipschitz continuous on bounded sets. A solution of problem $(3.5)$ in $[0, T]$, is a function

$$
w \in C\left([0, T], E_{\vartheta-1}\right) \cap C^{1}\left((0, T], E_{\vartheta-1}\right) \cap C\left((0, T], E_{\vartheta}\right)
$$


that satisfies (3.5) pointwise. We now introduce the concept of weak solutions for our semilinear problem. Let $\vartheta \in I_{q}$. We define the Dirichlet form associated to $\left(\mathcal{A}, \mathcal{B}_{1}\right)$ by

$$
a(\varphi, u):=\left\langle\partial_{i} \varphi, a_{i j} \partial_{j} u\right\rangle+\left\langle\varphi, a_{i} \partial_{i} u+a_{0} u\right\rangle+\left\langle\mu_{1} \varphi, \mu_{1} u\right\rangle_{1},
$$

for all $(\varphi, u) \in H_{q^{\prime}}^{2(1-\vartheta)}(\Omega) \times H_{q}^{2 \vartheta}(\Omega)$. Then,

$$
a \in \mathcal{L}^{2}\left(H_{q^{\prime}}^{2(1-\vartheta)}(\Omega) \times H_{q}^{2 \vartheta}(\Omega), \mathbb{R}\right) .
$$

Given a subinterval $J$ of $[0, T]$, with $0 \in J$, we set $\dot{J}:=J \backslash\{0\}$. Let $s=2 \vartheta$. We call $u$ a weak $H_{p}^{s}(\Omega)$-solution of (3.1)-(3.2) in $J$, if $u \in C\left(J, H_{p}^{s}(\Omega)\right)$ and

$$
\begin{aligned}
\int_{0}^{T^{\prime}} & \left\{-\left\langle\frac{d}{d t}\left(\varphi, \nu \mu_{1} \varphi\right),\left(u, \nu \mu_{1} u\right)\right\rangle+a(\varphi, u)\right\} d t \\
= & \int_{0}^{T^{\prime}}\left\{\langle\varphi, \hat{f}(t, u)\rangle+\left\langle\mu_{1} \varphi, \hat{g}(t, u)\right\rangle_{1} d t\right\}+\left\langle\varphi(0), u_{0}\right\rangle+\left\langle\left(\mu_{1} \varphi\right)(0), \mu_{1} u_{0}\right\rangle_{1}
\end{aligned}
$$

for all $T^{\prime} \in \dot{J}, \varphi \in C\left(\left[0, T^{\prime}\right], H_{q^{\prime}}^{2-s}(\Omega)\right)$ satisfying $\varphi\left(T^{\prime}\right)=0$, and $\left(\varphi, \mu_{1} \varphi\right) \in$ $C^{1}\left(\left[0, T^{\prime}\right], E_{-\vartheta}^{\sharp}\right)$.

Now define the projection $P: E_{\vartheta} \rightarrow H_{q}^{2 \vartheta}(\Omega), P(u, v):=u$. We are now ready to state our main existence and uniqueness result:

Theorem 3.1. Suppose that $\vartheta, \eta \in I_{q}$ satisfy $\eta<\vartheta$. Set $\xi:=1-(\vartheta-\eta)$. Then, given any $u_{0} \in H_{q}^{2 \vartheta}(\Omega)$, setting $w_{0}:=\left(u_{0}, \mu_{1} u_{0}\right)$,

(1) problem (3.5) has a unique maximal solution $w:=w\left(\cdot, w_{0}\right)$ satisfying

$$
w \in C\left(J, E_{\vartheta}\right) \cap C^{1-\xi}\left(J, E_{\eta}\right),
$$

and this solution, for all $t \in J$, satisfies the variation of constants formula

$$
w(t)=e^{-\mathbb{A}_{\vartheta-1} t} w_{0}+\int_{0}^{t} e^{-\mathbb{A}_{\eta-1}(t-\tau)} G(w(\tau), \tau) d \tau,
$$

(2) if $u:=P w$, then $u$ is the unique maximal weak $H_{q}^{s}(\Omega)$-solution of (3.1),

(3) the maximal interval of existence $J:=J\left(u_{0}\right)$ is open in $[0, T]$, and $u\left(\cdot, u_{0}\right)$ exists globally, provided $u\left(J, u_{0}\right)$ is bounded in $H_{q}^{s}(\Omega)$,

(4) in the autonomous case, i.e., in the case that $f$ and $g$ are independent of $t, u(\cdot, \cdot)$ defines a local semiflow on the phase space $H_{q}^{s}(\Omega)$, such that, $t \mapsto u(t, x)$ is a mapping from $J$ into $H_{q}^{s}(\Omega)$. Furthermore, that semiflow is such that bounded orbits are relatively compact.

For our problem (2.8), we can prove a global existence result, say, choosing $q=2$.

Theorem 3.2. For all $\vartheta_{0} \in H_{2, \mu_{2}}^{1}$ such that $0 \leq \vartheta_{0}(z) \leq \pi / 2$, the solution of (2.8) $\vartheta\left(\cdot, \vartheta_{0}\right) \in H_{2, \mu_{2}}^{1}$ is defined for all $t>0$. 
We outline the proof: Since in this case we are working in the classical Sobolev space $W_{\mu_{2}}^{1,2}$, the fact that all nonlinearities are globally bounded ensures that the free energy (2.6) immediately provides the required a priori $W^{1,2}$ estimates on the solutions.

\section{Multiplicity and Stability of Steady States}

\subsection{Linearisation around the vertical state}

Consider an equilibrium solution $\tilde{u}(x)$. The linearised problem about the solution $\tilde{u}$ is

$$
\left\{\begin{aligned}
v_{\tau} & =v_{x x}+2 \sigma \eta^{2} \cos (2 \tilde{u}) v, & & \text { for }(\tau, x) \in \mathbb{R}^{+} \times(0,1), \\
\nu v_{\tau} & =v_{x}-(4 \beta \cos (4 \tilde{u})+2 \kappa \eta \cos (2 \tilde{u})) v, & & \text { for }(\tau, x) \in \mathbb{R}^{+} \times\{0\}, \\
v & =0, & & \text { for }(\tau, x) \in \mathbb{R}^{+} \times\{1\}, \\
v & =v_{0}, & & \text { for }(\tau, x) \in\{0\} \times(0,1) .
\end{aligned}\right.
$$

This can be taken as the linear Cauchy problem

$$
v_{t}+A_{0} v=0, \quad v(0)=v_{0},
$$

where $A_{0} v:=\left(-v_{x x}+\omega_{1} v,-\nu^{-1} v_{x}(0)+\omega_{2} v(0)\right)$, for obvious definitions of $\omega_{1}$ and $\omega_{2}$, with $D\left(A_{0}\right)=\left\{(v, v(0)): v \in C^{2}(0,1) \cap C^{1}[0,1) \cap C[0,1], v(1)=0\right\}$.

The corresponding eigenvalue problem $\left(A_{0}+\lambda\right) \psi=0$, is thus

$$
\left\{\begin{array}{l}
-\psi_{x x}+\left(-2 \sigma \eta^{2} \cos (2 \tilde{u})+\lambda\right) \psi=0, \quad \text { for } x \in(0,1) \\
-\psi_{x}(0)+(4 \beta \cos (4 \tilde{u}(0))+2 \kappa \eta \cos (2 \tilde{u}(0))+\nu \lambda) \psi(0)=0 \\
\psi(1)=0
\end{array}\right.
$$

After making an obvious change of the dependent variable, this problem can be brought to a form so that we can apply the results in Ref. 5, taking into account that, in that work, the parameter $\lambda$ should be identified with our $-\lambda$. Therefore, (4.2) has infinitely many eigenvalues $\lambda_{n}, n=0,1, \ldots$ which are interlaced with the eigenvalues $\lambda_{n}^{D}$ of the Dirichlet problem obtained by taking $\psi(0)=0$. More explicitly: $\lambda_{0}>\lambda_{0}^{D}$ and $\lambda_{n} \in\left(\lambda_{n}^{D}, \lambda_{n-1}^{D}\right)$, for $n=1,2, \ldots$ Furthermore, each eigenvalue $\lambda_{n}$ is simple and its corresponding eigenfunction has exactly $n$ zeros in $(0,1)$. Reference 5 also provides some asymptotic estimates for $\lambda_{n}$, as $n \rightarrow \infty$ (see theorems and corollaries in Secs. 2, 3 as well as the remarks and results in Sec. 5 there).

We turn now our attention to the particular stationary solution $\psi(z) \equiv \frac{\pi}{2}$. The corresponding eigenvalue problem is

$$
\left\{\begin{array}{l}
-\psi_{x x}+\left(2 \sigma \eta^{2}+\lambda\right) \psi=0, \quad \text { for } x \in(0,1) \\
-\psi_{x}(0)+(4 \beta-2 \kappa \eta+\nu \lambda) \psi(0)=0 \\
\psi(1)=0
\end{array}\right.
$$


For this case, we can explicitly compute $\lambda_{n}^{D}=\lambda_{*}-(n+1)^{2} \pi^{2}, n=0,1, \ldots$, where $\lambda_{*}=-2 \sigma \eta^{2}$. We thus have:

Lemma 4.1. For the case $\sigma=+1$ :

If $\kappa \leq 1 / \sqrt{2}$, then $\lambda_{0}<0$;

If $\kappa>1 / \sqrt{2}$, then there is $\eta_{0}>0$ such that

$$
\eta<\eta_{0} \Rightarrow \lambda_{0}<0, \quad \eta=\eta_{0} \Rightarrow \lambda_{0}=0, \quad \eta>\eta_{0} \Rightarrow \lambda_{0}>0 .
$$

For the case $\sigma=-1$, there are $\eta_{-} \in(-\pi / \sqrt{2}, 0), \eta_{+} \in(0, \pi / \sqrt{2})$, such that

$$
\eta \in\left(\eta_{-}, \eta_{+}\right) \Rightarrow \lambda_{0}<0, \quad \eta \in\left\{\eta_{-}, \eta_{+}\right\} \Rightarrow \lambda_{0}=0,
$$

and $\lambda_{0}>0$, otherwise.

Proof. By writing $a(\lambda)=2 \sigma \eta^{2}+\lambda$ and $b(\lambda)=4 \beta-2 \kappa \eta+\nu \lambda$, (4.3) takes the form

$$
\left\{\begin{array}{l}
\psi_{x x}(x)-a(\lambda) \psi(x)=0 \quad \text { for } x \in(0,1) \\
\psi_{x}(0)-b(\lambda) \psi(0)=0 \\
\psi(1)=0
\end{array}\right.
$$

We now look for the relation between $a(\lambda)$ and $b(\lambda)$ for which this system has nontrivial solutions. By explicitly solving the differential equation with the condition at $x=1$ we find that, if $a(\lambda)>0$ then $\psi(x)=c \sinh (\sqrt{a(\lambda)}(x-1))$, if $a(\lambda)<0$ then $\psi(x)=c \sin (\sqrt{-a(\lambda)}(x-1))$, and if $a(\lambda)=0$ then $\psi(x)=c(x-1)$, where, in each case, $c \in \mathbb{R}$ is an arbitrary constant. Now we observe that conditions $a(\lambda)>0, a(\lambda)<0$ and $a(\lambda)=0$ are fulfilled if $\lambda>\lambda_{*}, \lambda<\lambda_{*}$ and $\lambda=\lambda_{*}$, respectively. Therefore, by imposing the boundary condition at $x=0$, there can only be a non-identically zero solution $\phi$ if and only if $b(\lambda)=g(\lambda)$ where

$$
g(\lambda)= \begin{cases}-\sqrt{a(\lambda)} \operatorname{coth} \sqrt{a(\lambda)}, & \text { if } \lambda>\lambda_{*} \\ -1, & \text { if } \lambda=\lambda_{*} \\ -\sqrt{-a(\lambda)} \cot \sqrt{-a(\lambda)}, & \text { if } \lambda<\lambda_{*}, \quad \lambda \notin\left\{\lambda_{0}^{D}, \lambda_{1}^{D}, \ldots\right\} .\end{cases}
$$

This function has vertical asymptotes at $\lambda=\lambda_{n}^{D}, n=0,1,2, \ldots$, is continuous and strictly decreasing in each interval contained in its domain, and $\lim _{\lambda \rightarrow+\infty} g(\lambda)=$ $-\infty$ (see Fig. 3).

An easy consequence of the definitions of $g$ and $b$ is the following:

(i) if $\lambda_{0}^{D}<0$, then

$$
\begin{gathered}
b(0)>g(0) \Rightarrow \lambda_{0}<0, \quad b(0)=g(0) \Rightarrow \lambda_{0}=0, \\
b(0)<g(0) \Rightarrow \lambda_{0}>0 ;
\end{gathered}
$$

(ii) if $\lambda_{0}^{D} \geq 0$ then $\lambda_{0}>0$. 


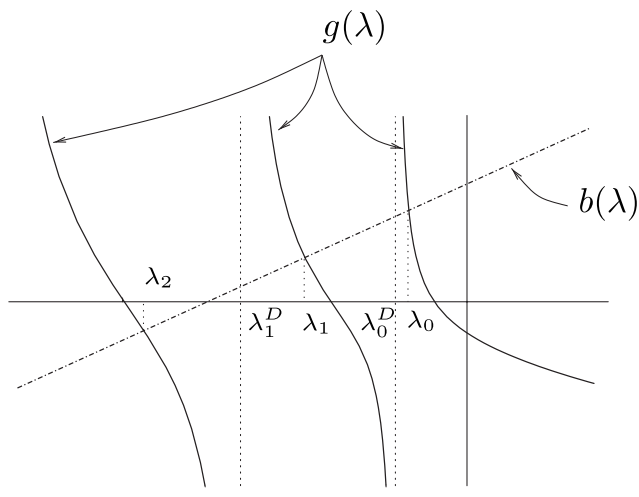

Fig. 3. Typical graphs of $b(\lambda)$ (dashed line) and of $g(\lambda)$ when $\lambda_{0}^{D}<0$.

Based on these results, we now study the sign of $\lambda_{0}$. Suppose first that $\sigma=+1$. Then, (i) occurs. Since $a(0)=2 \eta^{2} \geq 0$, then $g(0)=-\sqrt{2}|\eta| \operatorname{coth}(\sqrt{2}|\eta|)$. This expression defines a function $G(\eta)$ which is continuous for $\eta \neq 0$, has $\lim _{\eta \rightarrow 0} G(\eta)=$ -1 , is even, strictly concave, strictly decreasing for $\eta>0$ and has asymptotes $\eta \mapsto \pm \sqrt{2} \eta$. Since $b(0)=4 \beta-2 \kappa \eta$, we conclude that if $\kappa \leq 1 / \sqrt{2}$ then $b(0)>g(0)$ and therefore, $\lambda_{0}<0$, for all $\eta \in \mathbb{R}$. Otherwise $\kappa>1 / \sqrt{2}$, then there is $\eta_{0}>0$ such that, if $\eta=\eta_{0}$ then $\lambda_{0}=0$, if $\eta<\eta_{0}$ then $\lambda_{0}<0$, and if $\eta>\eta_{0}$ then $\lambda_{0}>0$.

Now take $\sigma=-1$. If $|\eta| \geq \pi / \sqrt{2}$ then case (ii) is observed and hence $\lambda_{0}>$ 0 . If $|\eta|<\pi / \sqrt{2}$, we are again in case (i) with $g(0)=-\sqrt{2}|\eta| \cot (\sqrt{2}|\eta|)$. This expression defines a function $H(\eta)$ for $|\eta|<\pi / \sqrt{2}$ which is continuous for $\eta \neq 0$, has $\lim _{\eta \rightarrow 0} H(\eta)=-1$, is even, strictly convex, strictly increasing for $\eta>0$ and has vertical asymptotes in $\eta= \pm \pi / \sqrt{2}$. Therefore, there are $\eta_{-} \in(-\pi / \sqrt{2}, 0)$ and $\eta_{+} \in(0, \pi / \sqrt{2})$ such that if $\eta \in\left(\eta_{-}, \eta_{+}\right)$, then $b(0)>g(0)$ and therefore $\lambda_{0}<0$; if $\eta \in\left(-\pi / \sqrt{2}, \eta_{-}\right) \cup\left(\eta_{+}, \pi / \sqrt{2}\right)$, then $b(0)<g(0)$, and therefore $\lambda_{0}>0$, and if $\eta \in\left\{\eta_{-}, \eta_{+}\right\}$, then $\lambda_{0}=0$.

\subsection{Phase-plane analysis of the non-linear system}

We consider the following stationary problem, associated with the dynamic problem (2.8),

$$
\left\{\begin{array}{l}
u_{x x}+\sigma \eta^{2} \sin (2 u)=0 \quad \text { for } x \in(0,1), \\
u_{x}(0)-\beta \sin (4 u(0))-\kappa \eta \sin (2 u(0))=0, \\
u(1)=\frac{\pi}{2} .
\end{array}\right.
$$

We want to understand how the solution set of (4.4) changes as we change the applied electric field $\eta$. An immediate difficulty arises from the fact that $\eta$ enters both the differential equation of (4.4) and the boundary condition at $x=0$. To 
overcome this problem it is convenient to change variables $x \mapsto y:=|\eta| x$, so that the phase portrait becomes independent of $E$. But this requires the case $\eta=0$ to be treated separately.

Actually, the case $\eta=0$ has already been treated in Ref. 8, and can be completely and easily studied due to its linear character. For the sake of completeness we briefly present it here using the same kind of analysis we will use for the $\eta \neq 0$ cases.

Writing $v:=u_{x}$, the differential equation in (4.4), with $\eta=0$, becomes

$$
\left\{\begin{array}{l}
u_{x}=v, \\
v_{x}=0
\end{array}\right.
$$

whose orbits are straight lines parallel to the $u$-axis. Treating $x$ as a time-like variable, we see that the solutions to (4.4) are obtained by checking the points in the phase plane that lie on the "initial" manifold

$$
\tilde{\Lambda}_{0}:=\{(u, v): v=\beta \sin (4 u)\}
$$

and for which a solution $u\left(x ; 0, u_{0}, v_{0}\right)$ of $(4.5)$ with condition $\left(u_{0}, v_{0}\right) \in \tilde{\Lambda}_{0}$ at $x=0$ takes the value $\frac{\pi}{2}$ when $x=1$. To this end, define the isochrone $\Xi_{0}$ as the set of points $\left(u_{0}, v_{0}\right)$ for which solutions $u\left(x ; 0, u_{0}, v_{0}\right)$ of $(4.5)$ satisfy $u\left(1 ; 0, u_{0}, v_{0}\right)=\frac{\pi}{2}$. It is easy to conclude that $\Xi_{0}$ is the straight line

$$
\Xi_{0}:=\left\{\left(u_{0}, v_{0}\right): v_{0}=\frac{\pi}{2}-u_{0}\right\}
$$

and the sought for solutions are those whose initial data lie in $\tilde{\Lambda}_{0} \cap \Xi_{0}$ with $0<u_{0}<$ $\frac{\pi}{2}$. (If the anchoring on the bistable surface is sufficiently strong, it is also possible to obtain "super-tilted" states where a rotation of the director of greater than $\pi / 2$ occurs within the cell, say with $u_{0} \sim \pi / 2$. However, since such states will always be of higher energy than the basic states, we will always choose $0<u_{0}<\frac{\pi}{2}$.) This intersection is always nonempty provided $\beta \geq \hat{\beta}$, where $\hat{\beta}>\frac{1}{4}$ is the unique solution of $\pi+\arccos \frac{1}{4 \beta}=\sqrt{(4 \beta)^{2}-1}$, which is the equation obtained when the curves $\tilde{\Lambda}_{0}$ and $\Xi_{0}$ intersect at a single point. If $\beta>\hat{\beta}$ we have exactly two intersection points, the one with smaller value of $u_{0}$ is the initial value for the HAN solution.

We gather this information in the following lemma:

Lemma 4.2. If $\eta=0$ and $\beta$ is sufficiently large, the system of equations (4.4) has three stationary solutions in the order interval $[0, \pi / 2]$.

Consider now the cases with $\eta \neq 0$. As pointed out above, an analogous phase plane analysis requires the change of variables $x \mapsto y=|\eta| x$ in order to fix the phase portrait independently of $\eta$. With this new independent variable, system 


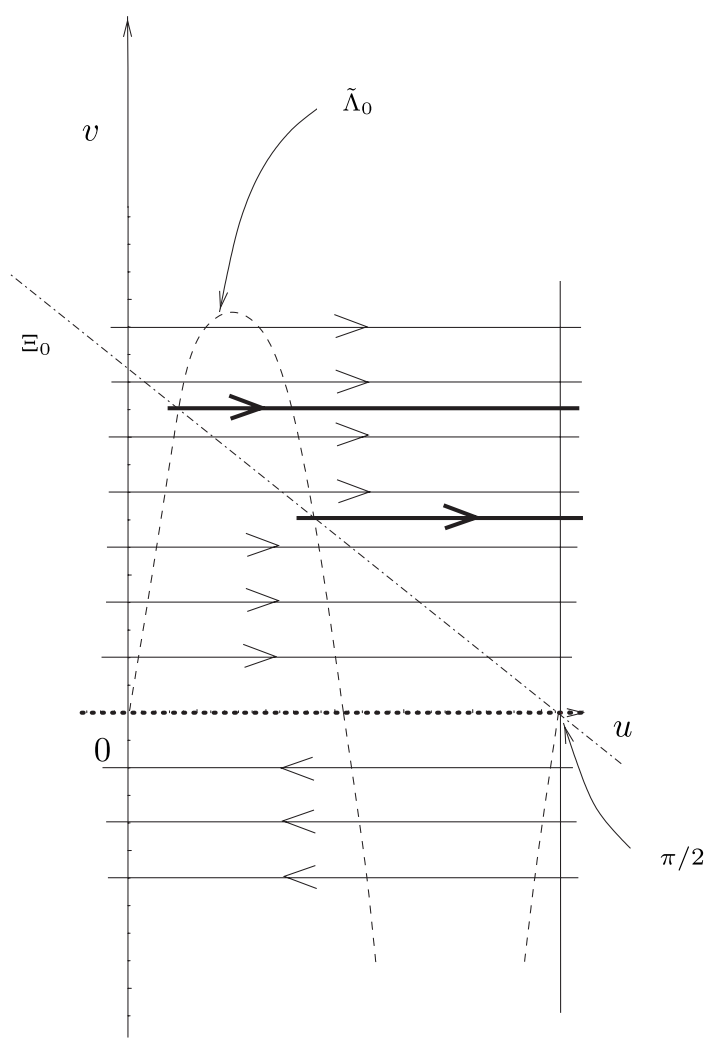

Fig. 4. Phase portrait in the case $\eta=0$.

(4.4) becomes

$$
\left\{\begin{array}{l}
u_{y y}+\sigma \sin (2 u)=0, \quad y \in(0,|\eta|), \\
u_{y}(0)-\frac{\beta}{|\eta|} \sin (4 u(0))-\kappa \operatorname{sgn}(\eta) \sin (2 u(0))=0 \\
u(|\eta|)=\frac{\pi}{2}
\end{array}\right.
$$

Define the isochrone $\Xi_{\eta}$ for (4.8) analogously to what was done for $\Xi_{0}$. Now, of course, $\Xi_{\eta}$ will not be a straight line (see below). Let $\Lambda_{\eta}$ be the initial $(y=0)$ manifold defined by

$$
\Lambda_{\eta}:=\left\{(u, v): v=\frac{\beta}{|\eta|} \sin (4 u)+\kappa \operatorname{sgn}(\eta) \sin (2 u)\right\} .
$$

Again, our analysis will be based on the study of the intersections between $\Xi_{\eta}$ and $\Lambda_{\eta}$. The geometry of the problem is distinctly different in the cases of positive and negative dielectric anisotropies and hence we must consider separately the situations of $\sigma=1$ and $\sigma=-1$. 
Case I: Let $\sigma=+1$. The first-order system equivalent to the differential equation in (4.8) is

$$
\left\{\begin{array}{l}
u_{y}=v \\
v_{y}=-\sin 2 u
\end{array}\right.
$$

This is the nonlinear pendulum equation with first integral $W(u, v)=v^{2}-\cos 2 u$. Hence, the isochrone $\Xi_{\eta}$ is the set of points $\left(u_{0}, v_{0}\right)$ of the phase plane satisfying

$$
\int_{u_{0}}^{\pi / 2} \frac{d \xi}{\sqrt{W\left(u_{0}, v_{0}\right)+\cos 2 \xi}}=|\eta| .
$$

It is clear from the phase plane analysis that the geometry of $\Xi_{\eta}$ is as shown in Fig. 5.

Also, for a fixed energy $W$, if $|\eta|$ increases $u_{0}$ must decrease, which implies that, as $|\eta| \rightarrow+\infty, \Xi_{\eta}$ converges (say in the Hausdorff metric) to the union of heteroclinic orbits connecting the saddle points $\left(\frac{2 n-1}{2} \pi, 0\right)$ to $\left(\frac{2 n+1}{2} \pi, 0\right)$, for $n \leq 0$. Call $\Xi_{\infty}$ the part of the heteroclinic from $\left(-\frac{\pi}{2}, 0\right)$ to $\left(\frac{\pi}{2}, 0\right)$ that lies in the first quadrant.

As in the case $\eta=0$, the solutions to (4.8) are in one-to-one correspondence with the points of $\Lambda_{\eta} \cap \Xi_{\eta}$.

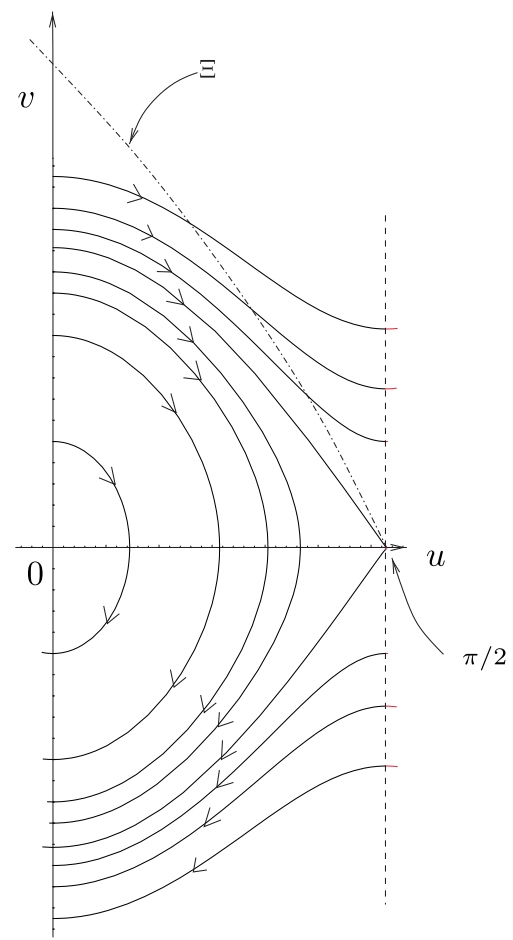

Fig. 5. Phase portrait and isochrone for $\sigma=+1$. 
There are essentially two distinct cases, namely $\kappa>\frac{1}{\sqrt{2}}$ and $\kappa<\frac{1}{\sqrt{2}}$, and the borderline case $\kappa=\frac{1}{\sqrt{2}}$. These situations match the linear stability analysis of the Vertical State solution, $u=\frac{\pi}{2}$, performed in Sec. 4 (see Lemma 4.1). From a geometrical point of view these two cases correspond to the possible distinct relative positions of $\Xi_{\eta}$ and $\Lambda_{\eta}$ when $\eta \rightarrow+\infty$.

Define $\Lambda_{+\infty}:=\{(u, v): v=\kappa \sin 2 u\}, \Lambda_{-\infty}:=\{(u, v): v=-\kappa \sin 2 u\}$. Observe that these curves are symmetric upon reflection in the $u$ axis.

By linearisation of $(4.10)$, the slope of $\Xi_{\infty}$ at $\left(\frac{\pi}{2}, 0\right)$ can be easily concluded to be $-\sqrt{2}$. Furthermore, observe that the slope of $\Lambda_{+\infty}$ at the same point is equal to $-2 \kappa$.

Let us consider first $\kappa>\frac{1}{\sqrt{2}}$. In this case, at $\left(\frac{\pi}{2}, 0\right)$ the absolute value of the slope of $\Xi_{\infty}$ is smaller than that of $\Lambda_{+\infty}$. Hence, close to $\left(\frac{\pi}{2}, 0\right)$, the curve $\Xi_{\infty}$ is below $\Lambda_{+\infty}$. This implies the existence of a unique point in $\Lambda_{+\infty} \cap \Xi_{\infty}$ with $0<u<\frac{\pi}{2}$ and thus the same uniqueness result for $\Lambda_{\eta} \cap \Xi_{\eta}$ holds for all $\eta$ sufficiently large. By decreasing $\eta$ we thus obtain the situation depicted in Fig. 6 if $\beta>\hat{\beta}$. If $\beta<\hat{\beta}$ the only difference is that the turning point case occurring at $\eta=b<0$ now happens at a positive value of $\eta$, since in this situation the analysis of the case $\eta=0$ gives the single solution $u=\frac{\pi}{2}$.

The bifurcation point from the $\mathrm{V}$ solution is a pitchfork bifurcation; the other branch of (unstable) solutions, where $u>\pi / 2$, is not drawn: they correspond to decreasing solutions $(v<0)$ with $u_{0}>\frac{\pi}{2}$ and are located in the phase diagrams, relative to the equilibrium point $\left(\frac{\pi}{2}, 0\right)$, in a symmetric position to that of the branch shown in Fig. 6. The bifurcation point is at the value of $\eta$ for which the curves $\Lambda_{\eta}$

$$
\eta=a \ll 0
$$
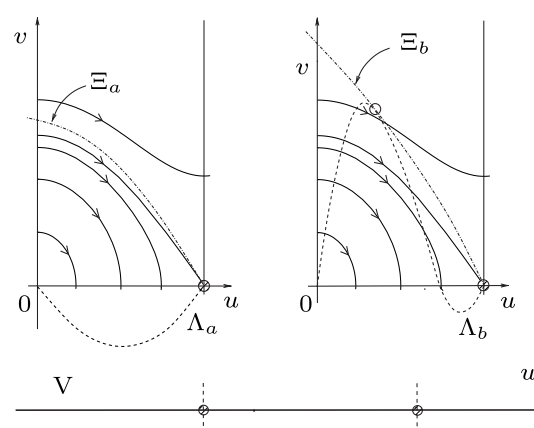

$\eta=b<0$

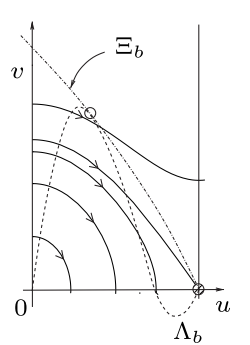

$\eta=c>0$

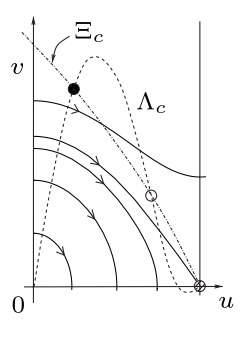

$\eta=d \gg 0$

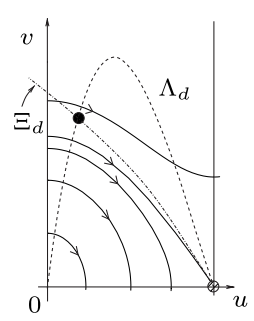

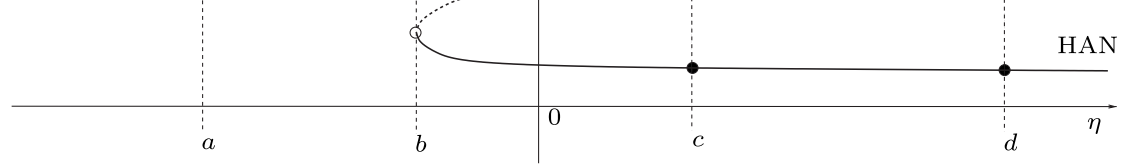

Fig. 6. Phase portraits and bifurcation diagram for $\sigma=+1$ and $\kappa>\frac{1}{\sqrt{2}}$. 
and $\Xi_{\eta}$ have equal slopes at $\left(\frac{\pi}{2}, 0\right)$. This value should obviously coincide with the value of $\eta$ for which the $\mathrm{V}$ solution loses stability, which was shown in Sec. 4.1 to be the unique positive solution $\eta_{0}$ of the equation

$$
4 \beta-2 \kappa \eta=-\sqrt{2} \eta \operatorname{coth}(\sqrt{2} \eta)
$$

From this equation, by plotting $\eta \mapsto \frac{4 \beta}{\eta}-2 \kappa$ and $\eta \mapsto-\sqrt{2} \operatorname{coth}(\sqrt{2} \eta)$, we immediately observe that $\eta_{0}=\eta_{0}(\kappa) \uparrow+\infty$ as $\kappa \downarrow \frac{1}{\sqrt{2}}$. The limit case, when $\kappa=\frac{1}{\sqrt{2}}$, correspond geometrically to the situation when the slopes of $\Xi_{\infty}$ and $\Lambda_{+\infty}$ at the $\mathrm{V}$ solution are equal. The analysis, following the same pattern as in the previous case, results in the bifurcation diagram presented in Fig. 7.

Let us now consider the case $\kappa<\frac{1}{\sqrt{2}}$. Since now, at the $\mathrm{V}$ solution, the absolute value of the slope of $\Xi_{\infty}$ is larger than that of $\Lambda_{+\infty}$ we conclude that $\Xi_{\infty}$ is above $\Lambda_{+\infty}$ for the whole region $0<u<\frac{\pi}{2}$, and so no solution exist for all $\eta$ sufficiently large. Decreasing the value of $\eta$ we conclude that, in this region of $u, \Lambda_{\eta} \cap \Xi_{\eta}$ is nonempty provided $\beta$ is sufficiently large. Thus we obtain the situation depicted in Fig. 8.

It is geometrically clear that the turning points in the bifurcation diagram in this figure occur at those values of $\eta$ for which there is a single intersection between $\Lambda_{\eta}$ and $\Xi_{\eta}$ (both curves with the same slope at the intersection point). Observe that in this case the $\mathrm{V}$ solution does not lose stability, and no switching happens from $\mathrm{V}$ to $\mathrm{HAN}$, although a HAN state does exist for a bounded range of values of $\eta$.

$$
\eta=a \ll 0
$$
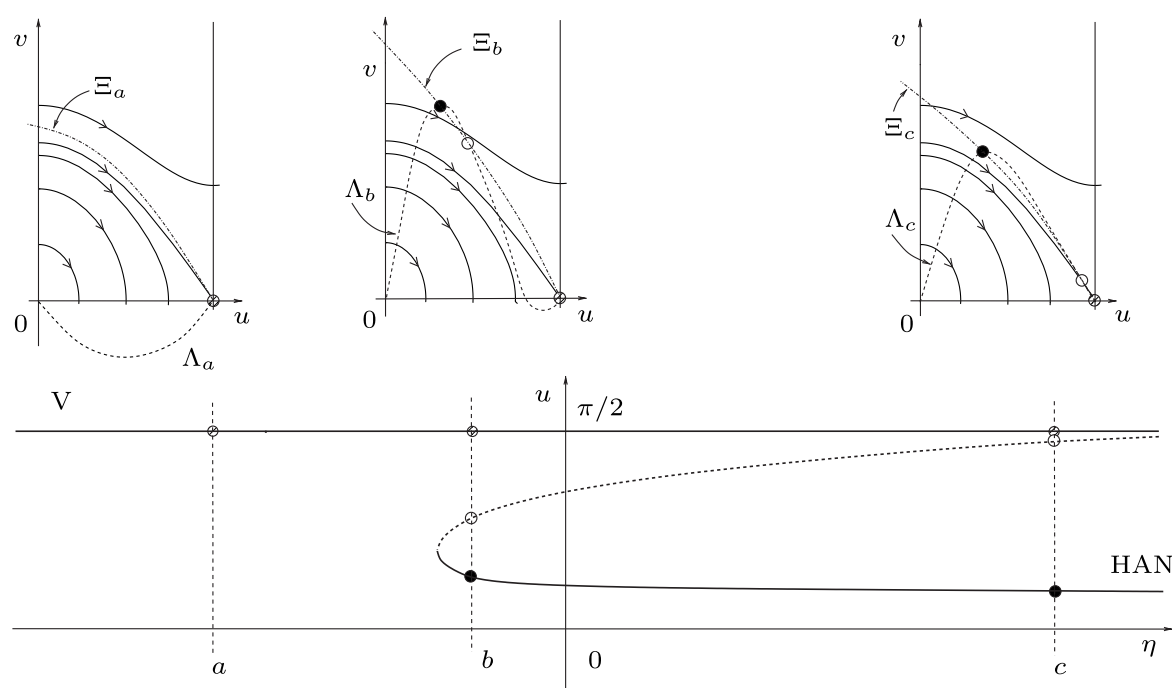

Fig. 7. Phase portraits and bifurcation diagram for $\sigma=+1$ and $\kappa=\frac{1}{\sqrt{2}}$. 


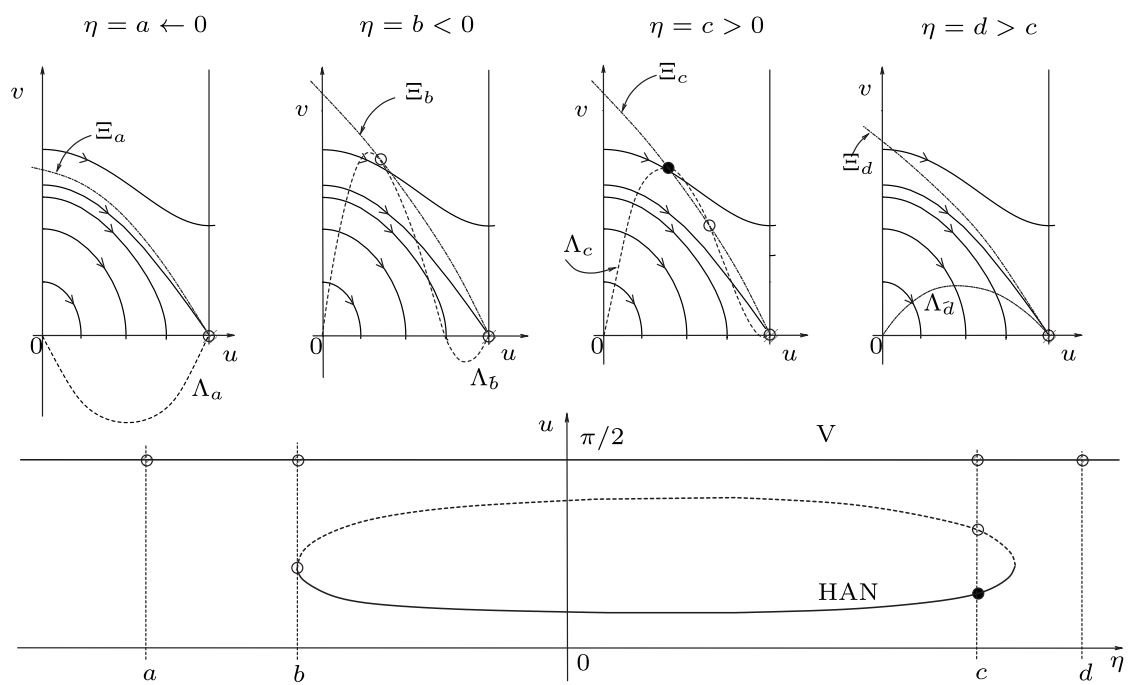

Fig. 8. Phase portraits and bifurcation diagram for $\sigma=+1$ and $\kappa<\frac{1}{\sqrt{2}}$.

Considering the above results in terms of the physical parameters of the system, we formulate the following theorem:

Theorem 4.1. For sufficiently large anchoring strength $W_{0}$ and average flexoelectric coefficient $E_{13}$, as is the case in Fig. 6, there are values $\underline{E}<0<\bar{E}$ such that in the order interval $u \in[0, \pi / 2]$ the $V$ solution is locally asymptotically stable for all $E<\bar{E}$ while the $H A N$ solution is locally asymptotically stable for all $E>\underline{E}$.

Therefore, if we are on the $\mathrm{V}$ solution branch and increase the strength of the electric field beyond $\bar{E}$ and return it to zero (slowly enough), we will effect the jump from $\mathrm{V}$ to HAN; decreasing it beyond $\underline{E}$ and returning to zero will cause the device to switch from HAN to $\mathrm{V}$.

Proof. By Lemma 4.1, for sufficiently large $E_{13}$ we are in the case $\kappa>1 / \sqrt{2}$ and the $\mathrm{V}$ solution will be linearly stable for all values of $\eta$ smaller than some $\bar{\eta}>0$, that is, for all $E$ smaller than some value $\bar{E}>0$. If $\beta$ is large enough, that is, if the anchoring strength $W_{0}$ is large enough, we have shown above that the HAN solution exists for values of $\eta>\underline{\eta}$, where $\underline{\eta}<0$; in other words, the HAN solution exists for all values of $E$ larger than some value $\underline{E}<0$. This disposes of the multiplicity part of the proof; it remains to deal with the stability of the HAN solution.

First, we can appeal to a principle of linearised stability for problems of the form of (3.1) proved by Merino. ${ }^{17}$ Finally, we employ a Conley index argument to show that the HAN solution is l.a.s for $E>\underline{E}$. For general background on the Conley index, see Ref. 19; arguments similar to the ones used here can also be found also in Ref. 13. We compute the (homology) Conley index of HAN as follows: Let $S=\operatorname{inv}([0, \pi / 2])$. Then $M_{1}$ (the V solution) and $M_{2}$ (see Fig. 9) are adjacent 


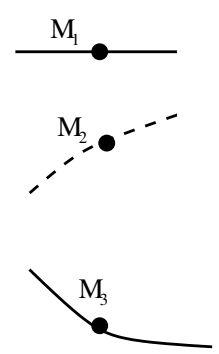

Fig. 9. The Morse decomposition.

under the flow defined order. Since $M_{1}$ is an attractor by Lemma 4.1 and since by analysing a neighbourhood of the pitchfork bifurcation from the $\mathrm{V}$ solution, it can be shown that $h\left(M_{2}\right)=\Sigma^{1}$, the connection matrix corresponding to the Morse decomposition $\left(M_{1}, M_{2}\right), M_{1}<M_{2}$ is

$$
C_{1}=\left(\begin{array}{ll}
0 & 1 \\
0 & 0
\end{array}\right)
$$

Now, since the interval $\left(M_{2}, M_{3}\right)$ continues to the empty set and since the system is dissipative, we have either (A) $\left(M_{2}, M_{3}\right)$ is a Morse decomposition with $M_{2}<M_{3}$ having the connection matrix

$$
C_{2}=\left(\begin{array}{ll}
0 & 1 \\
0 & 0
\end{array}\right)
$$

or (B) $\left(M_{3}, M_{2}\right)$ is a Morse decomposition with $M_{3}<M_{2}$ and the connection matrix

$$
C_{3}=\left(\begin{array}{ll}
0 & 1 \\
0 & 0
\end{array}\right)
$$

Case (A) would mean that $h\left(M_{3}\right)=\Sigma^{2}$ (and we would have for free a heteroclinic connection from $M_{3}$ to $M_{2}$; in case (B) we would have $h\left(M_{3}\right)=\overline{1}$ (and a heteroclinic connection from $M_{2}$ to $M_{3}$ ).

Let us show that case (A) is not possible. If it were, the connection matrix for the Morse decomposition $\left(M_{1}, M_{2}, M_{3}\right), M_{1}<M_{2}<M_{3}$, would have been

$$
C=\left(\begin{array}{lll}
0 & 1 & 0 \\
0 & 0 & 1 \\
0 & 0 & 0
\end{array}\right) \text {. }
$$

But this is not a connection matrix, since $C \circ C \neq 0$ (it is also rank 2, while we need rank 1). Note that in case (B) for the Morse decomposition $\left(M_{1}, M_{3}, M_{2}\right)$, $M_{1}<M_{2}, M_{3}<M_{2}$ we get

$$
C=\left(\begin{array}{lll}
0 & 0 & 1 \\
0 & 0 & 1 \\
0 & 0 & 0
\end{array}\right),
$$

which indeed squares to zero and has rank 1. 
Thus $h\left(M_{3}\right)=\overline{1}$, i.e. it is an attractor in $S$ (and it remains so for all $E>0$ by remaining isolated and the invariance of the index).

Case II. Now let us consider the case $\sigma=-1$. A complete analysis, such as that given for $\sigma=+1$ is not possible here for the reasons we explain below, but one can still prove a switching theorem similar to Theorem 4.1. More precisely, we have

Theorem 4.2. For sufficiently large $W_{0}$ and $E_{13}$, in the order interval $u \in[0, \pi / 2]$ we have that:

(a) The $V$ (constant) solution is locally asymptotically stable for $E \in\left(E_{1}, \bar{E}\right)$, where $E_{1}<0<\bar{E}$; at $\bar{E}$ it loses stability in a subcritical pitchfork bifurcation; at $E_{1}$ it exchanges stability in a supercritical pitchfork bifurcation to a non-monotone stationary solution which we will denote by $u_{n m}$.

(b) There is a value of $E, \underline{E}<0$ such that for all $E>\bar{E}$ there exists a locally asymptotically stable HAN solution of (2.7).

(c) For each value of E, there are at most two l.a.s. stationary solutions.

Before we prove this theorem, let us make a number of relevant remarks.

1. It is difficult to establish the exact ordering between $\underline{E}$ and $E_{1}$. If $E_{1}<\underline{E}$, the switch as $E$ is decreased is between the HAN and the V solutions; however, if $E_{1}>\underline{E}$ the switch is from the HAN to the non-monotone solution $u_{n m}$; see Fig. 11 for clarification and Fig. 5 of Ref. 8 for pictures of $u_{n m}$. In both cases we would expect the system to return along the $\mathrm{V}$ solution branch as $E$ is returned to zero, provided $E$ is reduced sufficiently slowly.

2. The supercritical pitchfork alluded to in part (a) of the theorem is the first one in an infinite series of such bifurcations as we decrease $E$; however, beyond the first one, none of the bifurcations creates a stationary solution in the order interval $[0, \pi / 2]$.

Proof. Going back to the non-dimensionalised system (2.8), we note that the difficulty in this case stems from the fact that the isochrones corresponding to different values of $\eta$ do not admit a simple ordering; furthermore, these curves cannot be represented for large enough $|\eta|$ as graphs of $v$ over $u$. The reason is clear if we realise that now the phase-portrait of (4.8) is that of a nonlinear pendulum shifted by $\pi / 2$, so that the rest point $(0,0)$ is now a saddle-point while $(\pi / 2,0)$ is a centre. If we make the change of variables as in the derivation of $(4.8)$, we see that the isochrones start to wind around the point $(\pi / 2,0)$. However, since we are interested only in the stationary solutions in the order interval $[0, \pi / 2]$, we restrict ourselves to a portion of the isochrone $\Xi_{\eta}, \Xi_{\eta}^{\prime}$ defined as follows: if we parametrise the isochrone by the parameter $s$, so that $(u(0), v(0))=(\pi / 2,0)$, and $s_{0}(\eta)$ is the value of the parameter for which the isochrone intersects the line $u=\pi / 2$ for the last time (note that $v\left(s_{0}\right) \leq 0$ necessarily), then

$$
\Xi_{\eta}^{\prime}=\left\{(u(s), v(s)) \mid s \geq s_{0}(\eta)\right\} .
$$


Then it is clear that $\Xi_{\eta}^{\prime}$ defines again $v$ as a function of $u$, and that the graphs of these functions, again defined on the domain $(-\infty, \pi / 2)$ are ordered in $|\eta|$.

Now, if we consider situation for $\eta$ large, the geometry of $\Xi_{\eta}^{\prime}$ is easily described. Inside the region bounded by heteroclinic orbits, it lies close to the one corresponding to $v<0$ and intersects the one corresponding to $v>0$ once. It is monotone for all values of $s>s_{1}(\eta)$, where $v\left(s_{1}(\eta)\right)=0$ (there is only one such value); see Fig. 11.

Once the geometry of $\Xi_{\eta}^{\prime}$ is established it is easy to conclude that

(i) for large positive $\eta$ in addition to the $\mathrm{V}$ solution, $u=\pi / 2$, in the order interval $[0, \pi / 2]$ there is a unique monotone stationary solution; this is the HAN solution.

(ii) for large negative $\eta$ in addition to the $\mathrm{V}$ solution, in the order interval $[0, \pi / 2]$ there is a unique stationary solution. Since it corresponds to an intersection of $\Xi_{\eta}^{\prime}$ and $\Lambda_{\eta}$ for $v<0$, this solution is non-monotone but unimodal; we denote it by $u_{n m, \eta}$. See Fig. 10 .

To finish the multiplicity proof, we need to connect the information gathered above with the situation for $\eta=0$ given by Lemma 4.2. Arguing by continuity, monotonicity of $\Xi_{\eta}^{\prime}$ and the symmetries of the phase plane we conclude that the

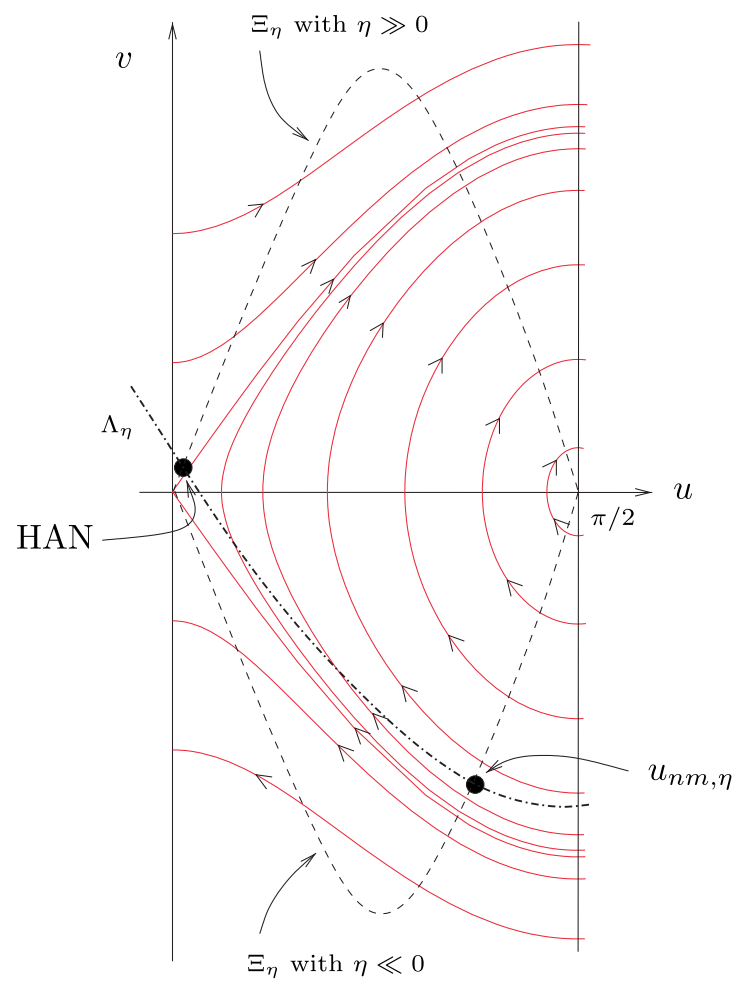

Fig. 10. Stationary solutions for large $|\eta|$. 
branch of $u_{n m, \eta}$ solutions bifurcates from the $\mathrm{V}$ solution in a pitchfork bifurcation for $\eta<0$, while for $\eta>0$ the $\mathrm{V}$ solution loses stability through a pitchfork bifurcation as well, which in view of the situation for large positive $\eta$ has to be a subcritical one, giving rise to the unstable monotone solutions. The question is whether these can be continued to $\eta=0$ to disappear at some value of $\eta<0$ in a saddle-node collision with the HAN solution branch. In theory it could happen that the solutions that exist for $\eta=0$ form an isola that does not connect to this branch. We now show that this cannot happen.

For $\eta>0$, consider (4.9), which defines $v$ as a function of $u$,

$$
v=V(u):=\frac{\beta}{|\eta|} \sin (4 u)+\kappa \sin (2 u) .
$$

Clearly, $V(\pi / 8)>\beta /|\eta|$. Now let us consider the "time" $x$ it takes the point $(\pi / 8, V(\pi / 8))$ to reach the line $u=\pi / 2$ under the flow of (4.8) with $\sigma=-1$. This distance $T$ is given by

$$
T=\int_{\pi / 8}^{\pi / 2} \frac{d \xi}{\sqrt{V(\pi / 8)^{2}+\cos (\pi / 4)-\cos (2 \xi)}} .
$$

Now note that

$$
\cos (\pi / 4)-\cos (2 \xi) \geq 0 \text { for all } \xi \in(\pi / 8, \pi / 2)
$$

and hence

$$
V(\pi / 8)^{2}+\cos (\pi / 4)-\cos (2 \xi)>\frac{\beta^{2}}{\eta^{2}}
$$

But then

$$
T<\frac{3 \pi|\eta|}{8 \beta},
$$

or in other words, for $\beta$ large enough we have that $T<|\eta|$ and this means that the point $(\pi / 8, V(\pi / 8))$ lies strictly above the isochrone $\Xi_{\eta}^{\prime}$ for all $\eta>0$. From the properties of the isochrone and the graph of $V(u), \Lambda_{\eta}$, it follows that these two curves have to intersect for some value $u<\pi / 8$. The implicit function theorem and Lemma 4.2 then ensure that the branches connect.

The stability of the $\mathrm{V}$ solution can be derived from Lemma 4.1; as in the case of $\sigma=+1$, this fixes the dimensions of unstable manifolds of all other equilibria and we have proved part (c) of the theorem. All the information is summarised in Fig. 11.

Case III. Finally, when $\sigma=0$ the differential equation in (4.4) is equivalent to (4.5) and so the isochrone $\Xi_{0}$ is still given by (4.7). The "initial" manifold $\Lambda_{\eta}$ is now

$$
\Lambda_{\eta}:=\{(u, v): v=\beta \sin (4 u)+\kappa \eta \sin (2 u)\}
$$

and the analysis proceeds as in Case I $(\sigma=+1)$ with $\kappa>1 / \sqrt{2}$ (and the phase portrait changed to the paralel flow shown in Fig. 4 resulting in a bifurcation diagram similar to that in Fig. 6. 


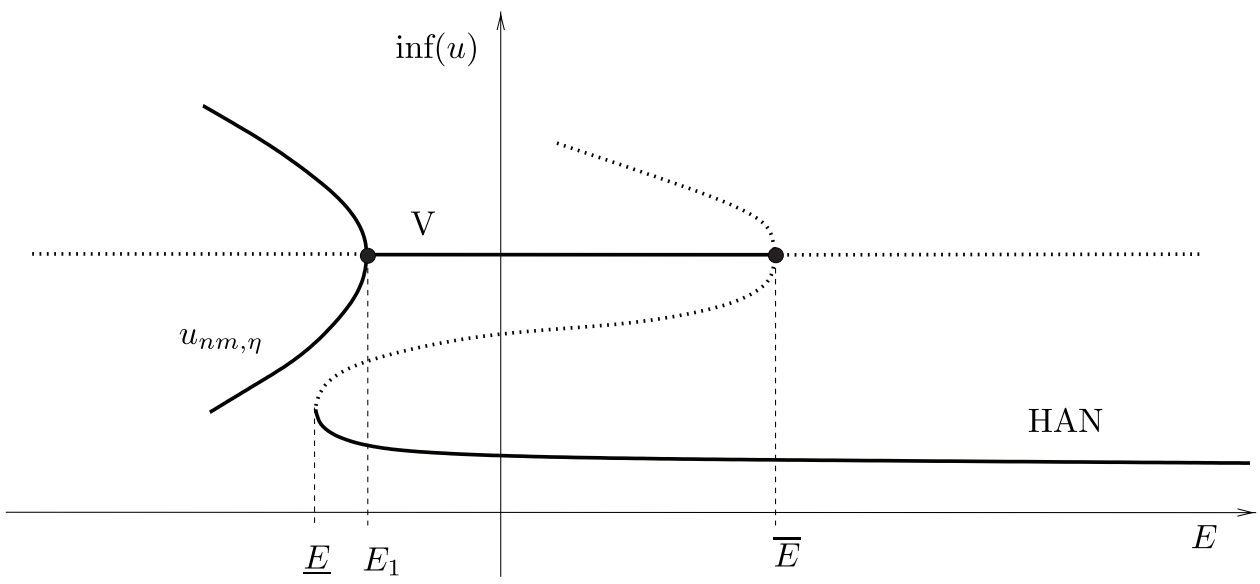

Fig. 11. Bifurcation diagram for $\sigma=-1$, large enough $\beta$ and $\kappa$.

\section{High-Frequency Excitation}

Another interesting question concerns the states that can be accessed by applying a high frequency AC voltage. To answer this question, we consider (2.8) with $\eta:=$ $\eta_{m} \sin (\tau / \epsilon)$. We have the following result for $\sigma=+1$ :

Theorem 5.1. Consider (2.8) with $\eta=\eta_{m} \sin (\tau / \epsilon)$. Then for $\epsilon$ sufficiently small there is a $2 \pi \epsilon$-periodic locally asymptotically stable solution in $H^{1}$ close to the $V$ solution that never loses stability.

Proof. We are going to appeal to the averaging theorem for parabolic PDEs proved in Ref. 14. That theorem is applicable, since in view of Lemma 3.1(3), the linear part of (2.8) generates an analytic semigroup and the nonlinearity is Fréchetdifferentiable on $W^{1,2}$. Thus, we need to consider the rest points of the averaged system corresponding to (2.8) with $\eta=\eta_{m} \sin (\tau / \epsilon)$. Stable rest-points of the averaged system will correspond to stable $2 \pi \epsilon$-periodic solutions of the periodically excited system (2.8). The rest points of the averaged system satisfy

$$
0=u_{x x}+\frac{1}{2} \sigma \eta_{m}^{2} \sin (2 u), \quad x \in(0,1)
$$

subject to the averaged boundary conditions,

$$
u_{x}(0)-\beta \sin [4 u(0)]=0, \quad u(1)=\frac{\pi}{2} .
$$

Clearly, as the flexoelectric effect has been averaged out, there is no mechanism for the V solution to lose stability; see Lemma 4.1. The conclusion is that even if a HAN-like time-periodic solution exists, it is not accessible from a neighbourhood of the V-like solution.

In the case of $\sigma=-1$, a similar argument shows that one cannot switch from a HAN solution to a Vertical one. 


\section{Conclusions}

In summary we will revert to dimensional variables in order to examine the significance of these results.

The successful operation of a bistable liquid crystal device relies, in part, on two fundamental principles. The first that there are (at least) two locally stable liquid crystal director configurations which exist without an applied electric field, and the second that it is possible to switch between these states using an applied electric field.

The results in this paper show that in order to achieve two stable states when the electric field $E$ is zero, the bistable surface anchoring strength must be sufficiently large (Lemma 4.2).

Given that we do indeed have two stable zero electric field states, we have also shown that for materials with a positive dielectric anisotropy, $\Delta \epsilon>0(\sigma=+1)$, then in order to be able to switch to and from the Vertical and HAN states the flexoelectric coefficients must be sufficiently large. Specifically $e_{11}+e_{33}>\sqrt{\epsilon_{0} \Delta \epsilon K}$ $(\kappa>1 / \sqrt{2})$ (Theorem 4.1).

For a material with a negative dielectric anisotropy, $\Delta \epsilon<0(\sigma=-1)$, it is also necessary to have sufficiently large flexoelectric cofficients to switch between states. However, in this case it is possible that a large negative electric field will induce a non-monotonic solution which will only switch back to the Vertical state if the electric field is increased from a large negative value to zero sufficiently slowly, since otherwise it may be possible to jump from the $u_{n m}$ solution to the HAN, so that the $\mathrm{V}$ solution is not accessed (Theorem 4.2).

Finally, we have also shown that the application of a high-frequency electric field severely limits the operation of the device. For both positive and negative dielectric anisotropies $(\sigma>0, \sigma<0)$ it will only be possible to switch in one direction (HAN to Vertical or Vertical to HAN respectively) and the reverse switching will be impossible to achieve.

\section{Acknowledgments}

F.P. da C. and J.T.P. acknowledge partial funding by Fundação para a Ciência Tecnologia through project PDCT/MAT/56476/2004.

\section{References}

1. H. Amann, Nonhomogeneous linear and quasilinear elliptic and parabolic boundary value problems, in Function Spaces, Differential Operators and Nonlinear Analysis, Teubner Texte im Mathematik, Vol. 133 (1993), pp. 9-126.

2. H. Amann, Linear and Quasilinear Parabolic Problems, Vol. 1 : Abstract Linear Theory, Monographs in Mathematics (Birkhäuser, 1998).

3. J. M. Arrieta, P. Quittner and A. Rodríguez-Bernal, Parabolic problems with nonlinear dynamical boundary conditions and singular initial data, Diff. Integ. Eqs. 14 (2001) 1487-1510. 
4. J. Bergh and J. Löfström, Interpolation Spaces: An Introduction (Springer-Verlag, 1976).

5. P. A. Binding, P. J. Browne and B. A. Watson, Sturm-Liouville problems with eigenparameter dependent boundary conditions, Proc. Edin. Math. Soc. 37 (1993) 57-72.

6. C. V. Brown, G. P. Bryan-Brown and J. C. Jones, U.S. Patent No. 6,249,332 19 June 2001.

7. C. V. Brown and N. J. Mottram, Influence of flexoelectricity above the nematic Fréedericksz transition, Phys. Rev. E 68 (2003) 031702-01-031702-05.

8. A. J. Davidson and N. J. Mottram, Flexoelectric switching in a bistable nematic device, Phys. Rev. E 65 (2002) 051710-1-051710-10.

9. A. J. Davidson, Flexoelectric switching in a bistable nematic device, $\mathrm{PhD}$ thesis, University of Strathclyde (2004).

10. J. Escher, Quasilinear parabolic systems with dynamic boundary conditions, Commun. Partial Diff. Eqns. 18 (1993) 1309-1364.

11. J. Escher, On the qualitative behaviour of some semilinear parabolic problems, Diff. Integ. Eqs. 8 (1995) 247-267.

12. M. Fila and P. Quittner, Large time behavior of solutions of a semilinear parabolic equation with a nonlinear dynamical boundary condition, in Topics in Nonlinear Analysis, The Herbert Amann Anniversary Volume (Birkhäuser, 1998), pp. 251-272.

13. M. Grinfeld and A. Novick-Cohen, The viscous Cahn-Hilliard equation: Morse decomposition and structure of the global attractor, Trans. AMS 351 (1999) 2375-2406.

14. J. K. Hale and S. M. Verduyn-Lunel, Averaging in infinite dimensions, J. Integ. Eqns. Applic. 2 (1990) 463-494.

15. T. Hintermann, Evolution equations with dynamic boundary conditions, Proc. Roy. Soc. Edin. 113A (1989) 43-60.

16. S. Kitson, Controllable alignment of nematic liquid crystals around microscopic posts: Stabilization of multiple states, Appl. Phys. Lett. 80 (2002) 3635-3637.

17. S. Merino, Existence theory for a highly degenerate semilinear reaction-diffusion system with nonlinear boundary conditions, preprint, Heriot-Watt University, Edinburgh (1996).

18. S. Merino, M. Grinfeld and S. McKee, A degenerate reaction diffusion system modelling a biosensor, Z. Angew. Math. Phys. 49 (1998) 46-85.

19. K. Mischaikow, Conley index theory: Recent developments, Lect. Notes Math. Vol. 1609 (Springer-Verlag, 1995), pp. 119-207.

20. K. R. Rajagopal and A. R. Srinivasa, Modeling anisotropic fluids within the framework of bodies with multiple natural configurations, J. Non-Newt. Fluid Mech. 99 (2001) 109-124.

21. A. A. Sonin, Surface Physics of Liquid Crystals (Gordon \& Breach, 1995).

22. A. M. Sonnet, P. L. Maffettone and E. G. Virga, Continuum theory for nematic liquid crystals with tensorial order, J. Non-Newt. Fluid Mech. 119 (2004) 51-59.

23. I. W. Stewart, The Static and Dynamic Continuum Theory of Liquid Crystals: A Mathematical Introduction (Taylor \& Francis, 2003). 